\title{
Low Power Devices for an Electronic Adapter with Mitigating the Soft Errors
}

\author{
Vinothini $\mathrm{N}^{1}$, Naveen Kumar $\mathrm{D}^{2}$, Uma Maheswari $\mathrm{T}^{3}$, Kanimozhi $\mathrm{T}^{4}$ \\ $P G$ Scholar RVS CET, Dindigul ${ }^{1}$ \\ $P G$ Scholar Karpagam University ${ }^{2}$ \\ Assistant Professor/EEE, RVS CET, Dindigul ${ }^{3}$ \\ Assistant Professor/EEE, RVS CET, Dindigul ${ }^{4}$
}

\begin{abstract}
This work presents the implementation of a high efficiency adapter using voltage regulators along with the converters. A new structure of the slim-type processor is proposed, which is composed of copper wire as the primary winding and printed circuit- board winding on the outer layer as the secondary winding be layout drawn using a Cadence virtuoso tool and by the simulation process it is tested in a 180nm CMOS technology. The proposed structure is suitable for a slim and high-efficiency regulator as well as converter because it has advantages of easy utilization and wide conductive cross sectional area compared to the other works. In addition the voltage doublers rectifier is applied to the secondary side due to its simple structure of secondary winding, and a CLC filter is adopted to reduce the output filters size. The validity of this study is confirmed by the experimental results.
\end{abstract}

Index Terms: CLC output filter, slim-type transformer, and voltage-doubler rectifier.

\section{Introduction}

Household appliances must have a network processor inorder to communicate with other appliances through a homenetwork. This additional processor increases the cost of theappliance when it is embedded. In addition, thecommunication medium for a home network has notconverged to a unique standard at present [1], [2]. Therefore,there is a need for adapters that convert appliance data /messages to the correct home network format.Recently, the communication protocol between anappliance and an adapter has been standardized [2], the CACI(Compact Appliance Control Interface) was reported in [1].But now, it is necessary to develop adapters, because manyappliances still use proprietary communication protocols. Thispaper describes the adapter, which can downloadcommunication protocol conversion programs, and cancommonly be used for various appliances.In addition, household appliances must be able to increasepower supply capacity in order to supply electrical power tothe adapter. In this paper, we selected IEEE $802.11 \mathrm{~b}$ wirelessLAN as a communication medium for the home network; however, other types of networks are easily supported.Household appliances must be capable of supplying peakpower that enables transmitting or receiving $802.11 \mathrm{~b}$ frames.This peak power is about $1 \mathrm{~W}$, and the power save mode(transceiver is periodically in active) is the same as the normalmode (transceiver is always in active) [3], [4]. This paperdescribes how to reduce the peak power using the smoothingcircuit (SC) and the dynamic bandwidth controller (DBC).An outline of this paper is as follows, in section II webriefly describe the home network architecture in which ouradapter operates, section III introduces the softwarearchitecture of the adapter itself and details it's functionalities, section IV discusses power management issues whenconnecting the adapter to existing appliances. Section $\mathrm{V}_{\text {offers }}$ some concluding remarks.

\section{Review Of Methods}

\section{A. RT Method}

In the RT method [2], [3], one port of the adapter isconnected to a calibrated port of the VNA, and the otherport is terminated in a reflective load, as shown in Fig. 1.The reflection coefficient of the adapter and load is measuredat reference plane 2 , and the process is repeated with thereflective load replaced by a second reflective load, whosereflection coefficient differs in phase from that of the first. In practice, the reflective terminations are typically an offsetshort and an offset open for a coaxial port and a flush short andan offset short for a waveguide port. The relation between the reflection coefficients measured at reference plane 2 in Fig. 1and the intrinsic efficiency of the adapter was derived in [3].Assuming a reciprocal, low-loss adapter.

\section{Proposed Structure}

\section{A. Slim-Type Transformer}

The use of PCB trace as both the primary and the secondarywinding has been researched for the reduced height of thetransformer as shown in Fig. 2. Although this method canmake it easy to build the slim transformer, large portion ofthe PCB cannot be used for conduction since many turnsneed wide space for the 
insulation. Therefore, conduction loss can be increased due to the reduced conductive crosssectionalarea. Also, the high cost for the manufacturing of eachPCB winding can be the problem. To overcomes these drawbacks, the sandwiched or interleavingstructure using copper wire as the primary winding andPCB trace as the secondary winding was researched as shownin Fig. 2. The copper wire is more suitable than the PCBtrace for the plenty of turns in the primary side. However, itcannot use the enough window area of the slim transformer,considering the insulation between the primary and the secondarywinding. It is also difficult to utilize the spiral-woundcoil of the primary winding without a bobbin.

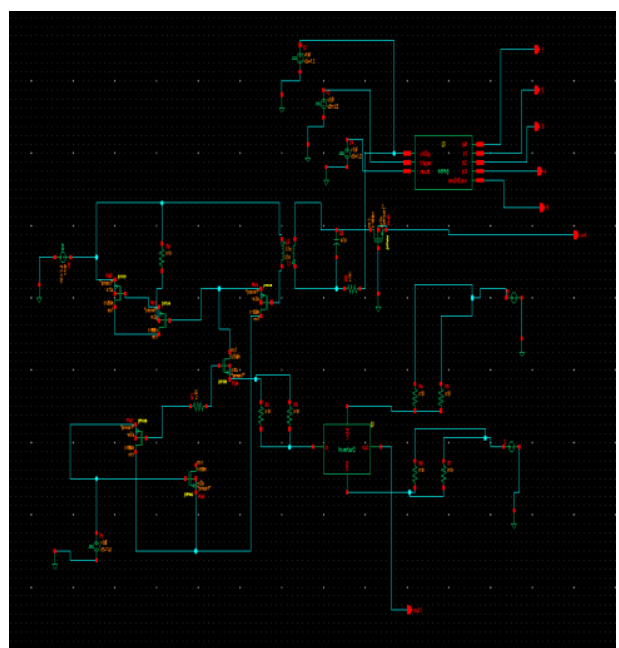

Fig.1. structure of the proposed scheme

The proposed structure of the slim-type transformer is composedof copper wire on the bobbin as the primary winding andPCB winding on the outer layer as the secondary winding. Inthe slim-type transformer, the insulation between the primaryand the secondary winding takes considerable portion of thewindow area when the windings are stacked as shown in Fig. 2 and 3. However, when the primary and secondarywindings are positioned inside and outside, respectively, thevolume of insulation between them can be greatly decreasedas shown in Fig. 2(c). The saved area can be contributed to theconductive cross-sectional area, and the conduction loss can bereduced. Also, the utilization of the primary winding is easierdue to the use of the bobbin.Additionally, the leakage inductance of the transformer is avery important parameter in the $L L C$ resonant converter sinceit is directly related to the resonance and this value can becontrolled by using this structure. The leakage inductance isdetermined by the distance between the primary and secondarywindings generally, and the distance can be designed fromthe PCB pattern. Therefore, the $L L C$ resonant converter canbe implemented without the need to add an external resonantinductor.

\section{Voltage-Doubler Rectifier and CLC Output Filter}

The topology selection of the secondary side is also veryimportant to build a high-efficiency and slimtype convertersince it determines the design of the transformer. As shown in Fig. 3, the center-tapped, fullbridge, and voltage-doubler rectifiers can be attractive candidates of the secondary-siderectifier. Among many characteristics, the configuration of thesecondary winding is very critical to the design of the slimtypetransformer since large current is flowing through thesecondary side and wide conductive area is needed. Assumethat the turns ratios of the transformer in the center-tapped, full bridge, and voltage-doubler rectifiers are $n p_{\_} c t: n s_{-} c t: n s_{-} c t, n p_{-} f b: n s_{\_} f b$, and $n p_{-} v d: n s_{-} v d$, respectively. When the primaryturns of aforementioned rectifiers are the same, $n s_{-} c t$ and $n s_{-} f b$ are designed twice $n s_{-} v d$ to obtain the same voltageconversion ratio.

Additionally, since the center-tapped rectifierconfiguration needs two different secondary windings, it is notappropriate to the slim-type transformer due to its complexstructure of the secondary PCB winding. In this point of view, the voltage-doubler rectifier can be selected for an optimal onewith the proposed transformer, which can be realized with justone turn in the secondary winding.Meanwhile, when the capacitor output filter is adopted, thecurrent flowing through the output capacitor is discontinuous. Therefore, the rms ripple current of the output capacitor isgenerated very large, and the bulky capacitors are needed tobear that. To reduce the output filter size in that case, a $C L C$ filter is widely used as shown in Fig. 4(a). Due to the additional small inductor, large current ripple is generated in the left-side capacitor $C f$, and the current ripple of the right-side capacitor $C O$ is very small. Thus, the multilayer ceramic capacitor is applied to $C f$ since it has high rms ripple current rating andsmall size, and the electrolytic capacitor is applied to $C O$ sinceit has large capacitance value for dynamic 
response. With the voltage-doubler rectifier, using the $C L C$ filter ismore attractive since the additional passive components areless than with other rectifiers. Because doubler capacitors, $C 1$ and $C 2$, are already in the voltagedoubler rectifier, only the additional small inductor is needed to achieve the $C L C$ filterconfiguration as shown in Fig. 4.

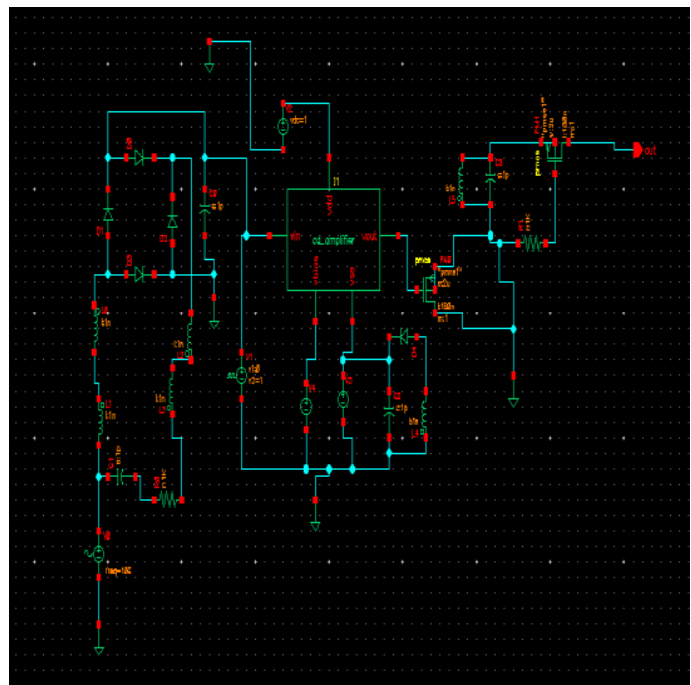

Fig.2.implemmenting the proposed adapter

\section{The Legacy System Challenge}

Legacy systems present a number of technical challenges toachieving net-centric standards compliance. These issues ofteninclude: software architectures [16], data formats, external interfaces,and constraints of safety and security. These challenges are discussed below.

\section{A. Legacy Software Architectures}

The typical legacy tactical system in DoD today was not designedto comply with net-centric technical standards in eithertheir internal software architectures or their external interfaces.The authors have observed that many of these systems were notdesigned to use some of the key Internet-related standards, suchas HTML, HTTP, SOAP, WSDL, and XML. The reasons for thisinclude: 1) the inability of Internet standards to satisfy real-time,mission critical requirements [12]-[18] and 2) the computingstandards and commercial products available at the time manyof today's military systems were developed [12]-[17].

The authors have observed two prevalent mechanisms usedfor communications within legacy military software architecturesby server and client software applications: a) Remote Procedure Calls (RPC) made using Application ProgrammingInterfaces (APIs), and b) the Common Object Request BrokerArchitecture (CORBA) [12]. Both RPC/APIs and CORBA usethe Transmission Control Protocol/Internet Protocol (TCP/IP)for message transmission.Fig. 1 depicts the use of RPC over socket-based APIs to supportcommunications between client and server software applicationsover a network. The figure shows a client application and a server application connected to a common network. The clientneeds to perform a function on some data, while the server canperform that function.To initiate this function call, the client uses an RPC toremotely call the server function. The client uses software"sockets" and TCP/IP to call the function. On the network, TCP/IP is used to transmit the function call and provide it onthe proper socket.

The server recognizes the RPC, performs thefunction, and provides the results back to the client. Fig. 2 depicts the use of CORBA to support communicationsbetween client and server software applications over a network.CORBA, a specification developed by the Object Management Group [19], is an object-oriented counterpart to a socket-basedAPI. In the object-oriented paradigm, the calling applicationinvokes a "method" on an object. An Object Request Broker (ORB), which is a software implementation of the CORBA. Specification keeps track of the locations of software andobjects on the network. It determines the location of ObjectA's implementation and invokes the appropriate method on thatobject.

\section{B. Legacy Data Formats and Access}

Data in a typical legacy system are held in legacy formats.Often these consist of custom and proprietary data formats andon venations that were selected by the developer to initiallyimplement each legacy system. Legacy formats may be due to the lack of data standards and/or the lack of interoperabilityrequirements at the time the system was developed. In addition, the legacy data is rarely accessible via standard, net- 
centricmechanisms (e.g., web services). Accessing and translatinglegacy data often requires a very large engineering effort.

\section{Legacy Point-to-Point Interfaces}

Traditionally in DoD, data exchange between two systemshas been defined for point-to-point interfaces [7]. These interfacesare numerous and essentially proprietary, because theyonly work for a pair of systems. On the other hand, net-centriccompliance requires a "many-to-many" data exchange approach [7]. This requires a more open approach to defining interfaces,as well as the implementation of net-centric technical standardssuch as XML.

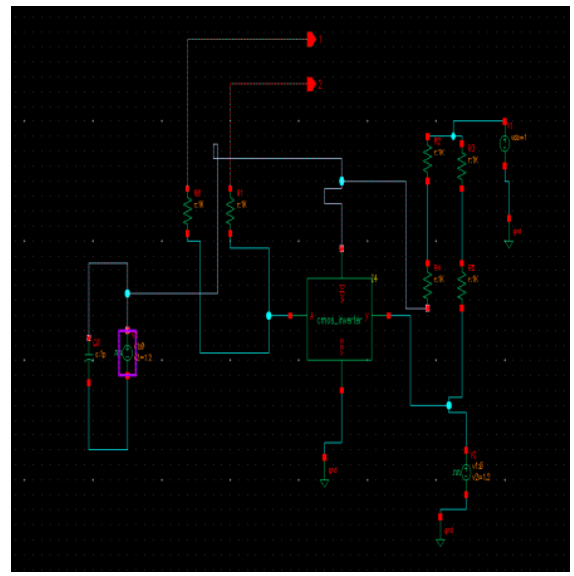

Fig.3. circuit for the proposed scheme operation cycle

\section{Safety and Security Constraints}

Legacy military systems typically have important safetyand security constraints on their design and performance. Forexample, weapon systems are concerned with weapon safetyrequirements, such as maintaining positive control of theirweapons under all circumstances. They may also be concernedwith issues such as crossing security domains when connectingto the GIG or with other systems with which interfaces arerequired. As an example, the authors have observed that thisis typical for systems on a ship, where a mixture of multiplesecurity domains exists, due to a variety of classification levels, access authorization, and "need to know."As a result, radically changing the software architectures ofsuch systems greatly increases risk and invites mission criticalfailure. Despite great advances in software technologies, currententerprise standards, tools and implementations are largelyunable to meet the most challenging real-time, deterministicrequirements of these critical systems [20]-[21].

\section{A. Net-Centric Adapter Concept}

\section{Net-Centric Adapter}

The goal of the Net-Centric Adapter for Legacy Systems (NCALS) is to enable legacy military systems to affordablyparticipate in a net-centric force. Specifically, NCALS minimizes the changes to legacy systems required to participate on the GIG. In addition, it is designed as a common, generic software component that could be used by many differentlegacy systems. To achieve this, several technical objective were necessary. Enable legacy systems to publish their data and services tothe GIG. Enable legacy systems to subscribe to GIG data and services. $\bullet$ Reduce the software development effort required to implement net-enabled legacy systems. $\bullet$ Architect the NCALS software as a common, configurable component. The NCALS software concept is illustrated in Fig. 3. It serves as an automated, two-way gateway between a legacy system and the GIG. As such, it works behind the scenes in an automated fashion to expose data from legacy systems to users of the GIG. In addition, it must be configurable, as a common software component, to support a variety of legacy systems needs, as well as portable across a variety of computing platforms.

Lastly, its architecturemust be scalable to accommodate the net-centric data requirements of many different legacy systems. A legacy system consists of legacy software components running on hardware, typically on a Local Area Network (LAN). These software components communicate with one another via legacy software interfaces (e.g., APIs and/or CORBA) and hold data in legacy formats. The NCALS software uses the existing legacy software interfaces to obtain legacy data, transform it into net-centric standard formats and publish it to the GIG in compliance with net-centric standards. Likewise, it transforms GIG data into legacy data formats and injects it into the legacy system via its existing software interfaces. Regardless of the particular domain, data and services of a legacy system, much of the NCALS functionality is common. However, it is 
configurable to accommodate the specifics of a particular legacy system operating in its specific domain ofoperations. Since NCALS is designed to enable net-centric operations, it focuses on enabling legacy systems to interoperate with the GIG. As a result, it provides a service-oriented architecture connection for a legacy system to the rest of the DoD enterprise on the GIG. However, it does not modify the legacy components to comply internally with the net-centric technical standards. It allows the legacy system architecture to remain largely undisturbed.

\section{Results}

The simulation results for the dialysis session without the adapterdemonstrate that the basic flow features are a high-speed jet (averageu-component of $270 \mathrm{~cm} / \mathrm{s}$ at the $\mathrm{x}$-location of the needle tip) issuingfrom the needle and a slower background vascular access flow (averageu-component of $60 \mathrm{~cm} / \mathrm{s}$ at the $\mathrm{x}$-location of the needle tip) within thegraft [Fig. 1]. Downstream of the needle tip, the needle flow impingesupon the bottom of the graft wall and begins to sweep upwardsalong the sides to the top of the graft, forming two large-scale helicalstructures that persist down the length of the graft. Immersed withinthese helical structures are a host of small-scale, complex, three-dimensionalflow structures that result in substantial flow unsteadinessdownstream of the dialysis needle. The graftwall experiences increasedhemodynamic stresses as a result of the impinging needle jet.

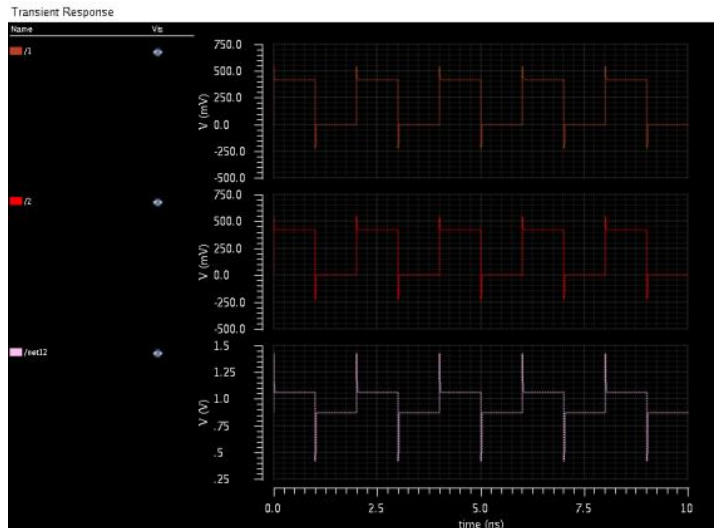

Fig.4. Transient analysis for the proposed scheme

This is evidenced by a sharp rise in the WSS [Fig. 2] field in the vicinityof the jet impingement region. Upstream of this region, the WSS isapproximately 30 dynes/cm2, but downstream of it, the WSS rapidlyundergoes an eightyfold increase to nearly 2400 dynes/cm2. When thefully deployed SMP adapter is present, the needle jet is oriented inthe downstream direction and no longer impinges as severely upon thegraft wall [Fig. 1]. As a result, the WSS [Fig. 2] is substantiallyreduced. In addition, the larger cross-sectional exit area of the adapter decelerates the needle flows. A comparison of the average u-componentof velocity (130 $\mathrm{cm} / \mathrm{s})$ of the needle flow at the distal tip of the adapterwith the average u-component of velocity $(70 \mathrm{~cm} / \mathrm{s})$ of the backgroundvascular access flow at the same x-location shows them to be closerin value, which could potentially reduce the strength of the shear layerinstability that develops downstream of the adapter.Images of the in vitro flow visualization prior to delivery of the SMPadapter are shown in Fig. 2and 3.

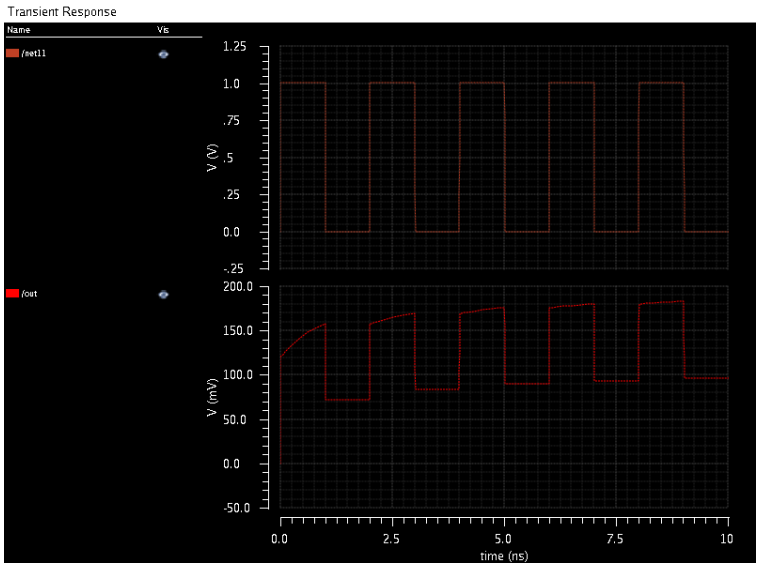

Fig.5. Transient analysis for the architecture 


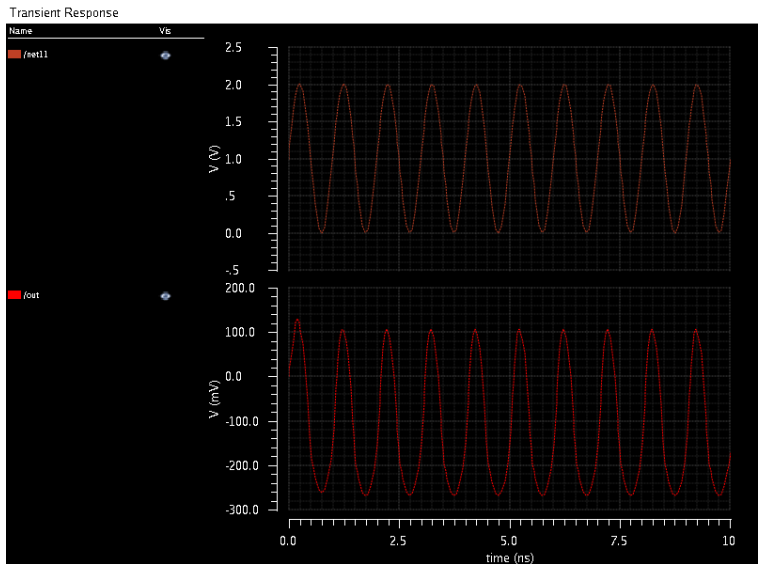

Fig.6. Transient analysis for the clocking cycle

Although the vascular access and needle flow rates and Reynolds numbers are well below the physiological values used in the CFD simulations, a qualitatively similar flow field is present in which the needle jet impinges upon the wall and wraps upward along the sides of the wall. After the compressed SMP adapter is delivered through the dialysis needle, the heated flow within the model graft actuates the adapter to its fully expanded shape [Fig. 5]. Complete actuation occurs within approximately $30 \mathrm{~s}$. Following the actuation, room temperature water $\left(\_21 \_C\right)$ is pumped through the graft model. The resulting flow visualization image [Fig. 3] with the deployed adapter demonstrates that the adapter directs the needle jet so that it is somewhat more aligned with the background vascular access flow.

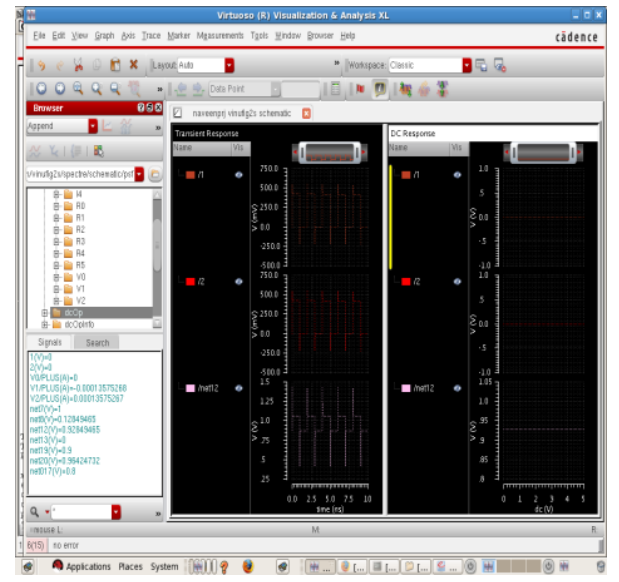

Fig.7. Transient analysis and DC analysis

\section{A. Monitoring of Load-Connection Block}

The MOLB monitors whether the load system is connectedor not. For this function, the MOLB generates $v \mathrm{CI}-\mathrm{ON}$ and sends it over to the CIOB when the load system is connected.The MOLB can be realized by using only one comparator andseveral resistors as shown in Fig. 2. When the load system is connected to the proposed adapter, since $v$ DET and $v \mathrm{CI}-\mathrm{ON}$ are "ON," optocouplers opto1 and opto2are turned ON. Then, the CIOB connects vlink to the HV pinof the control IC and $v$ CC to the VCC pin of the control IC.Consequently, the control IC starts its function. Each resistancecan be designed to satisfy (1). $R 3$ and $R 4$ can be designedconsidering the operating current of opto1 and opto2.

Table I:

\begin{tabular}{|l|l|}
\hline Part & Name \\
\hline Diode & GBLA07 \\
\hline Resistor & $1 \mathrm{M} \mathrm{OHMS}$ \\
\hline Capacitor & $250 \mathrm{nF}$ \\
\hline Link capacitor & $200 \mathrm{uF}$ \\
\hline Supply voltage & $3 \mathrm{~V}$ \\
\hline Output voltage & $1.8 \mathrm{~V}$ \\
\hline Power & $0.8 \mathrm{~mW}$ \\
\hline Current & $1.2 \mathrm{~A}$ \\
\hline
\end{tabular}


Table II:

\begin{tabular}{|c|c|c|c|}
\hline & This paper & [1] & [2] \\
\hline Area $\mathrm{mm}^{2}$ & 20 & 26 & 23 \\
\hline $\begin{array}{l}\text { Power } \quad(\mathrm{mW}) \\
\begin{cases}\mathrm{x} & 10\}\end{cases} \end{array}$ & 10 & 16 & 23 \\
\hline Current (mA) & 8 & 10 & 12 \\
\hline Voltage (V) & 3 & 5 & 4 \\
\hline
\end{tabular}

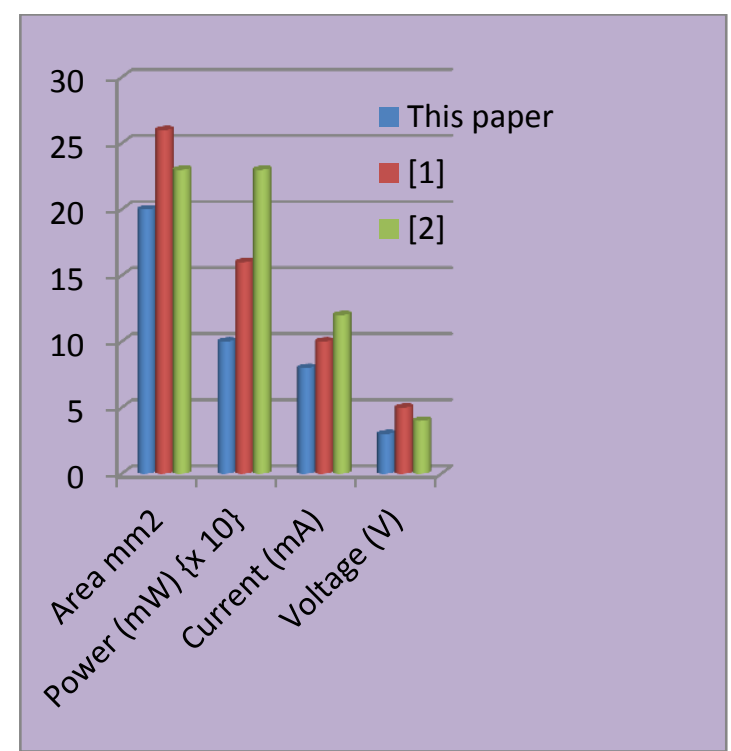

Fig.8. Comparative chart

\section{Discussion And Conclusion}

Through this initial investigation we have presented the results of anovel architecture for the newly designed for the low voltage level operations, dialysis needle adapter. The simulations demonstratethat the adapter significantly reduces the power and the area, which could potentiallyreduce thegeneral subsequent and subsequent vascular access occlusions. Thesuccessful preliminary in vitro test indicates that the concept of deliveringand actuating a SMP adapter through a dialysis needle has meritand should be further investigated and improved. It is evident that theprototype adapter does not entirely direct the flow in the downstreamdirection. This can be remedied by casting a longer regulators and adapter abouta mandrel that has an inherently curved shape. Given the ease of machiningand molding the material this and other modificationscan readily be incorporated into alternate adapter design. This be implemented in the Cadence Virtuoso tool of 180nm CMOS process.

\section{References}

[1] Byoung-Hee Lee and Gun-Woo Moon," Zero No-Load Power AC/DC Adapter for Electronic Equipment WithEmbedded Battery", IEEE transactions on power electronics, vol. 28, no. 7, July 2013.

[2] Research and Markets, External ac-dc power supplies: Worldwide forecasts, 10th ed., Apr. 2011.

[3] Joint Staff, An Evolving Joint Perspective: U.S. Joint Warfare and Crisis Resolution in the 21st Century Jan. $28,2003$.

[4] The White House, National Security Strategy of the United States of America Mar. 2006.

[5] OSD, Quadrennial Defense Review (QDR) Rep. Feb. 6, 2006.

[6] Chairman of the Joint Chiefs of Staff, National Military Strategy of the United States of America: A Strategy for Today; A Vision for Tomorrow 2004.

[7] DoD CIO, DoD Net-Centric Data Strategy May 9, 2003.

[8] SPAWAR Systems Center Charleston, FORCEnet Compliance Category Report Mar. 21, 2005.

[9] A. J. Simon, "Overview of the department of defense net-centric datastrategy," Crosstalk: J. Defense Softw. Eng., Jul. 2006.

[10] OASD (NII)/CIO, Net Centric Attributes List Feb. 17, 2004

[11] DoD IT Standards Registry (DISR) [Online]. Available: https://disronline.disa.mil/a/DISR/index.jsp, Jun. 16, 2008

[12] J. Gray and J. Valdivia, "Inter-LAN socket connection manager," inPoster, IEEE Int. Conf. Technologies for Homeland Security, May 12-13, 2008.

[13] A. Shalloway and J. R. Trott, Design Patterns Explained: A New Perspective on Object-Oriented Design. Reading, MA: AddisonWesley, 2002.

[14] G. Hohpe and B. Woolf, Enterprise Integration Patterns. Reading, MA: Addison-Wesley, 2004.

[15] D. Cozart-Amos, S. Soderlund, A. Thomas, and T. Turner, Commercial Off-The-Shelf (COTS) Product Evaluation Report for NetCentric Adapter for Legacy Systems (NCALS) Feb. 13, 2007, Internal NSWCDD Report.

[16] G. Lewis, E. Morris, and D. Smith, "Analyzing the reuse potential of migrating legacy components to a service-oriented architecture," in Proc. Conf. Software Maintenance and Reengineering 2006 (CSMR'06), Mar. 22-24, 2006, IEEE Computer Society. 
[17] F. Criste, Director, Communications Programs, OASD(NII)-DASD(C3, Space \& IT Programs), Presentation: "Implementing the Global Information Grid (GIG): A Foundation for 2010 Net Centric Warfare (NCW)" May 18, 2004.

[18] U.S. Department of Defense, “Global Information Grid,” sec. 7.2,Defense Acquisition Guidebook Dec. 16, 2004.

[19] U.S. Department of Defense (DoD) Chief Information Officer (CIO), Department of Defense Global Information Grid Architectural Vision: Vision for a Net-Centric, Service-Oriented DoD Enterprise ver. 1.0, Jun. 2007.

[20] L. Whitt, "Why is C4I software hard to develop?," in Proc. 12th Int.Command and Control Research and Technology Symp., Newport, RI, Jun. 2007.

[21] K. Lund, A. Eggen, D. Hadzic, T. Harfsoe, and F. T. Johnsen, Norwegian Defence Research Establishment, "Using web services to realize service oriented architecture in military communications networks,"IEEE Commun. Mag., vol. 45, no. 10, pp. 47-53, Oct. 2007.

[22] C. Kohlhof and R. Steele, "Evaluating SOAP for high-performancebusiness applications: Real-time trading systems," in Proc. 2003 Int.WWW Conf., Budapest, Hungary.

[23] R. van Engelen, "Pushing the SOAP Envelope with Web Services for Scientific Computing," in Proc. Int. Conf. Web Services, Erfurt, Germany, 2003.

[24] R. A. van Engelen, "Constructing finite state automata for highperformance web services," in IEEE Int. Conf. Web Services, Erfurt, Germany, 2004.[25] Q. Duan, "Service-oriented network description and discovery for highperformancegrid computing," inProc.IEEEInt. Conf. Service-Oriented Computing and Applications, Newport Beach, CA, 2007.
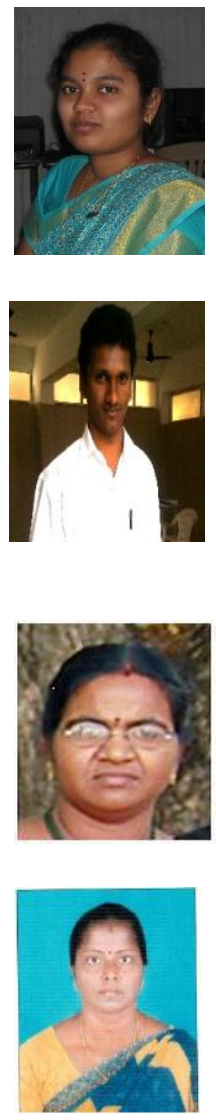

Vinothini Narayanan ${ }^{1}$ received the Bachelor of Engineering in Electrical and Electronics Engineering from P.S.N.A. College of Engineering \& Technology., Dindigul, Anna University Chennai in 2010.Currently doing Master of Engineering in Embedded Systems in RVS CET, Dindigul and worked as a Lecturer in K.Ramakrishnan College of Engineering in Trichy for the period of two years. And have published research papers in International Journal and International Conference. Her research area includes control systems, Embedded Automotive Networking with CAN, power electronics, embedded systems.

Naveen Kumar $\mathbf{D}^{2}$ received the Bachelor of Engineering in Electrical and Electronics Engineering from V.L.B.Janakiammal college of Engineering and Technology,Coimbatore, Anna university of Technology, Coimbatore in 2011. Currently doing Master of Engineering in VLSI Design in Karpagam University, Coimbatore. And have published two research papers in International Journals. His research interests include VLSI design inlow power, Clustering techniques in low power.

Uma Maheswari $\mathbf{T}^{3}$ received the Bachelor of Engineering in Electrical and Electronics Engineering from P.S.N.A. College of Engineering \& Technology., Dindigul in 2004 and received Master of Engineering in Power Electronics \& Drives from P.S.N.A. CET, Dindigul in 2008. And currently working as a Assistant Professor in RVS CET, Dindigul for past five years. Her research area includes power electronics, and power system, power quality and High voltage engineering.

Kanimozhi $\mathbf{T}^{4}$ received the Bachelor of Engineering in Electrical and Electronics Engineering from Alagappa Chettiar College of Engineering. \& Technology, Karaikudi in 2001 and M.Sc., Power Engineering from Nanyang Technological University, Singapore in 2009. And currently working as a Assistant Professor in RVS CET, Dindigul for past one year and four months. Having total experience of 2 years 11 months. Her research area includes control systems, power electronics, instrumentation and power system. 